Journal of Engineering and Applied Sciences 14 (Special Issue 9): 10487-10489, 2019

ISSN: 1816-949X

(C) Medwell Journals, 2019

\title{
Management of Formal and Informal Care of People with Dementia-Situation in the Czech Republic
}

\author{
${ }^{1}$ Petra Maresova, ${ }^{1}$ Blanka Klimova and ${ }^{1,2}$ Kamil Kuca \\ ${ }^{1}$ Faculty of Informatics and Management, University of Hradec Kralove, \\ Rokitanskeho 62, Hradec Kralove 3, 50003 Králové, Czech Republic \\ ${ }^{2}$ Biomedical Research Centrum, University Hospital Hradec Kralove, \\ Hradec Kralove, Czech Republic
}

\begin{abstract}
Dementia represents a significant burden for the economic systems of developed countries. The reason is an increasing trend of a number of elderly people aged 65 and 80 years and specifically these years are critical for the incidence of dementia since this disease rises with aging. The purpose of this study is to specify the economic burden of care of people with dementia in the area of formal and informal care. This study particularly focuses on the direct costs which include treatment, drugs, formal and informal care. The study concentrates on the European countries where the issue of aging population and provision of care about the people with dementia is very topical nowadays. This study also presents a case study, individual items of costs in one of the countries of central Europe-Czech Republic where the burden is expected to be higher than in many western countries. The methods include a method of literature review, analysis of web pages of day care centers and institutional facilities in the European countries with the purpose to obtain information about the costs of formal care. The costs specifications are based on Drummond's methodology and the costs are divided into direct and indirect. The result shows that the treatment costs are 2,393.14 EUR, annual costs of formal care are 5,403 EUR on average and annual costs of informal care are 9,326.4 EUR in the Czech Republic.
\end{abstract}

Key words: Costs, expenditure, formal care, informal care, dementia, population

\section{INTRODUCTION}

The international statistical classification of diseases and related health problems (WHO., 2016) defines dementia as a syndrome caused by the brain disease usually of chronic or progressive nature, during which many higher nerve cortical functions are damaged (WHO., 2016). Dementia represents a significant burden for the economic systems of developed countries (Maresova et al., 2015; Mohelska et al., 2015). Therefore, the purpose of this study is to specify the economic burden of care of people with dementia. This study focuses on the direct costs which include treatment, drugs, formal and informal care.

According to the data from the SHARE project, 21\% (in France and Switzerland) to 43\% (Czech Republic) of the non-institutionalized population of 65 years of age and above receive help or support at least sometimes on an informal basis. Of the population group aged 80 years and above, $41 \%$ receive informal support (in France, the Netherlands, Spain and Switzerland) rising to 60\% (Czech Republic) (Riedel and Kraus, 2011). Thus, this study concentrates on the European countries where the issue of aging population and provision of care about the people with dementia is very topical nowadays. This study also includes a case study, individual items of costs in one of the countries of Central Europe-Czech Republic.

\section{MATERIALS AND METHODS}

The methods include a method of literature review of the studies exploring the issues of formal and informal care of the people with dementia, analysis of web pages of day care centers and institutional facilities in the Czech Republic, analysis of Czech Statistical Office database (CSO) with the purpose to obtain information about the costs of formal care on 1.1. 2016, analysis of type of treatment with use data from the University Hospital in Hradec Kralove available on 30.6.2015.

The specification of the economic burden is based on Drummond's division of costs (Drummond et al., 1997). The costs are divided into two categories-direct and indirect costs. This study mainly focuses on the costs of treatment and formal and informal care which are part of the direct costs.

According to Triantafillou et al. (2010), informal care is characterized as follows:

\section{Corresponding Author: Petra Maresova, Faculty of Informatics and Management, University of Hradec Kralove,} Rokitanskeho 62, Hradec Kralove 3, 50003 Králové, Czech Republic 
Table 1: Costs of formal care in the Czech Republic

\begin{tabular}{ll}
\hline Institutional care price per day (EUR) & Parameters \\
\hline Establishment 1 (Karvina, 2015) & $12.25 /$ per day (including accommodation and food) \\
Establishment 2 (Alzheimer Centrum, 2015) & $17.4 /$ per day (including accommodation and food) \\
& Additional costs: Therapeutic dolls-148 \\
Establishment 3 [10] & Hirudotherapy (leeches)-37 EUR \\
Price per day/therapy & 13 EUR/day (including accommodation and food) \\
Establishment 1 (Libina, 2015) & \\
& Individual sessions (50-60 min) $=18.5$ EUR \\
Establishment 2 (Nadeje, 2015) & Group sessions (20-30 min) $=3.7-12.25$ EUR \\
\hline
\end{tabular}

Researchers

- Treatment predominantly provided by family members

- Carers are not professional caregivers

- Carers have no contracts with respect to care duties

- They do not receive any pay

Formal care is as follows:

- Services provided by the licensed professionals

- Services are controlled by the state or other types of organizations

- Caregivers receive a pay and they are entitled to social rights and working regulations

Costs of treatment, formal and informal care in the Czech Republic: As it has been already mentioned, the direct costs are specified by the costs on treatment and care, formal and informal care.

Treatment costs: The direct costs on ambulatory care in the Czech Republic per patient/per year are 99 EUR (Mohelska et al., 2015). These data were provided by the University Hospital in Hradec Kralove where patients visited their neurologist. This visit is held twice a year, according to the patient's state of health also three-four times a year, blood and cerebrospinal fluid samplings. In addition, the costs of drugs of 1, 776 EUR per year must be included (Leky, 2001). The total annual costs are then 1,875 EUR. From the inpatient care point of view, the length of hospitalization of the patients with a higher dementia degree ranged between 6,-5-15 days and average costs for the hospitalization were between 333-703 EUR, depending on the time of hospitalization. In the Czech Republic people with dementia are hospitalized in psychiatric centers and hospitals.

Costs of formal care: The costs of formal care range in the Czech Republic between 11-18.5 EUR per day including accommodation and food. Additional services must be paid. In case of day care centers which patients attend regularly in order to take the offered therapies, the prices range between 7.4-18.5 EUR. The important aspect is the fact whether the day care center also provides transport and food, individual or group care (Table 1). Other additional services include canistherapy,
Table 2: Amount of expenditure from the public budget on allowances for the dependent persons in the Czech Republic

\begin{tabular}{lr}
\hline Expenditure (mil EUR) & Values \\
\hline Degree of dependency (light dependency) & 36.02 \\
Degree of dependency (medium dependency) & 166.54 \\
Degree of dependency & 207.36 \\
Degree of dependency (full dependency) & 180.01 \\
\hline Own according to MPSV. (2015) &
\end{tabular}

Table 3: Amount of allowance on care in the Czech Republic. Allowance on care/monthly (EUR)

\begin{tabular}{lr}
\hline Allowance on care/monthly (EUR) & Values \\
\hline Degree of dependency (light dependency) & 29.61 \\
Degree of dependency (medium dependency) & 148.00 \\
Degree of dependency & 296.00 \\
Degree of dependency (full dependency) & 444.12 \\
\hline
\end{tabular}

Own according to MPSV. (2015)

ergotherapy, practicing fine motor skills, cognitive training, art therapy+music therapy, physical activities, walks and handicraft.

Costs of informal care: The costs of informal care mainly include caregiver's lost salaries. If a caregiver is a woman with mean age 55 years and mean hours of caring: 46h/week with secondary education, then in the Czech Republic an average salary of a person with secondary education is $777 \mathrm{EUR}$. The informal caregivers as well as institutionalized establishments can use a state allowance on care according to the age and the degree of dependency. In case of persons older than 18 years which are the patients with dementia, this allowance is between 36-180 mil. EUR for dependent person in the Czech Republic in the year of 2013 (Table 2). For individuals the amount of allowance is presented in Table 3.

\section{RESULTS AND DISUSSION}

Summary: A case study of the Czech Republic shows the costs of treatment, formal and informal care on the basis of the expenditure as follows:

Annual costs on treatment and drugs: Ambulatory care +average price of hospitalization $=1,875$ EUR+518.14 $\mathrm{EUR}=2,393.14 \mathrm{EUR}$. In addition, the fact is that every person with dementia does not have to report annual costs of hospitalization at all. 
Annual costs of formal care: Average price for the stay in the institutionalized establishments $=5,403$ EUR (between 3, 377.13 EUR -6, 754.26 EUR).

Annual costs of informal care: Monthly average lost caregivers' salaries $* 12=777.2 * 12=9,326.4$ EUR. In many cases the consequences and costs of informal care also include worsen psychic and physical health of caregivers.

From the percentage point of view, proportion of costs on treatment and drugs is formed by $43 \%$ of average costs of formal care and $25 \%$ of average costs of informal care.

\section{CONCLUSION}

The costs from the case study of the Czech Republic can be compared with other European countries. Nevertheless, the resulting values significantly influence the monitored and included items in the direct costs. The method of calculation of the total costs corresponds to the study conducted in France in which Gerves et al. (2014) presented that informal costs estimated with the opportunity cost method (e1,309-3,322) were higher than when estimated with the proxy good method (EUR 939- 2,407). Medical costs (out of which $60 \%$ relates to drugs) varied markedly according to patients, ranging from EUR410 for mild AD to EUR 622 for moderate to severe $\mathrm{AD}$. Informal care costs predominated in the share of total costs. These estimates are comparable to Kenisgsberg et al. (2009) findings which present annual direct costs of EUR9,914 per patient in France in 2008 (EUR 826 per month).

\section{ACKNOWLEDGEMENTS}

This study is published thanks to the support of the internal projects of University of Hradec Kralove: Economic and Managerial Aspects of Processes in Biomedicine.

\section{REFERENCES}

Alzheimer Centrum, 2015. Website of home for people with dementia. VU University Medical Center, Amsterdam, Netherlands. http://www.alzheimercentrum.cz/.
Drummond, M.F. and B.J. O’Brien, 1997. Methods for the Economic Evaluation of Health Care Programs. Oxford Medical Publication, Oxford, England,.

Gerves, C., P. Chauvin and M.M. Bellanger, 2014. Evaluation of full costs of care for patients with Alzheimer's disease in France: The predominant role of informal care. Health Policy, 116: 114-122.

Karvina, 2015. Website of home for the elderly. Karvina, Czech Republic. http://www.ndkarvina.cz/.

Kenisgsberg, P., K. Ersek, L. Gulacsi, K. Karpati and A. Jacques et al., 2009. Socio economic impact of Alzheimer type disease in Europe. Gerontology Soc., 128: 297-336.

Leky, 2001. Health and healthcare. Utery, Czech Republic. http://www.zdrav.cz/modules. php?op $=$ modload $\&$ name $=$ News $\&$ file $=$ article $\&$ sid $=$ 1635

Libina, 2015. Welcome to the website contributory organizations Social services Libina. Libina, Czech Republic. www.ddlibina.cz/.

MPSV., 2015. Preparing for aging. Fedral Ministry of Labour and Social Affairs, Taiwan. https://portal.mpsv.cz/soc/ssl/prispevek.

Maresova, P., H. Mohelska, J. Dolejs and K. Kuca, 2015. Socio-economic aspects of Alzheimer's disease. Curr. Alzheimer Res., 12: 903-911.

Mohelska, H., P. Maresova, M. Valis and K. Kuca, 2015. Alzheimer's disease and its treatment costs: Case study in the Czech Republic. Neuropsychiatric Dis. Treatment, 11: 2349-2354.

Nadeje, 2015. Registration for races run runczech for hope!. Nadeje, Czech Republic. http://www.nadeje.cz.

Riedel, M. and M. Kraus, 2011. Informal Care Provision in Europe: Regulation and Profile of Providers. European Network of Economic Policy Research Institutes, London,.

Triantafillou, J., M. Naiditch, R. Kvetoslava, K. Stiehr and S.N. Carretero et al., 2010. Informal care in the long-term care system. Interlinks, Vienna, Austria. http://interlinks.euro.centre.org/sites/default/files/ WP5_Overview_FINAL_04_11.pdf.

WHO., 2016. Multililingual and multipurpose. World Health Organization, Geneva, Switzerland. http://www.who.int/classifications/icd/en/. 